\title{
ON THE DEFINITION OF THE CONE SPECTRAL RADIUS
}

\author{
GUSTAF GRIPENBERG
}

(Communicated by Thomas Schlumprecht)

\begin{abstract}
For functions homogeneous of degree 1 and mapping a cone into itself two reasonable definitions of the cone spectral radius have been given. Although they have been shown to be equal in many cases, this note gives an example showing that the two definitions may differ for continuous, homogeneous of degree one functions which are also order-preserving in the partial ordering induced by the cone.
\end{abstract}

\section{INTRODUCTION}

The purpose of this note is to study under what assumptions two different definitions of the cone spectral radius of a function which is homogeneous of degree 1 and maps a cone $C$ in a normed linear space $X$ into itself can give different results. Our results in Section 3 answer a question which was raised in a remark on page 527 of 4 .

The Bonsall cone spectral radius of $f$ (introduced under a different name in [2]) is defined by

where $f^{k}=\underbrace{f \circ \ldots \circ f}_{k}$ and

$$
\tilde{r}_{C}(f)=\lim _{k \rightarrow \infty}\left\|f^{k}\right\|_{C}^{1 / k}
$$

$$
\left\|f^{k}\right\|_{C}=\sup \left\{\left\|f^{k}(x)\right\|_{X}: x \in C \text { and }\|x\|_{X} \leq 1\right\} .
$$

(It follows from the subadditivity of $\log \left(\left\|f^{k}\right\|_{C}\right)$ that the limit exists.)

In 4 the following definition is introduced as well:

$$
r_{C}(f)=\sup \left\{\limsup _{k \rightarrow \infty}\left\|f^{k}(x)\right\|_{X}^{1 / k}: x \in C\right\} .
$$

Clearly, one always has $r_{C}(f) \leq \tilde{r}_{C}(f)$. In [4, Theorems 2.2 and 2.3] and [5, Theorem 3.4] conditions are given under which $r_{C}(f)=\tilde{r}_{C}(f)$. See also [1] where a formula characterizing the growth rate of orbits is given and [3] where the continuity of the mapping $f \mapsto r_{C}(f)$ is studied.

The main purpose of this note is to give examples in which $r_{C}(f)<\tilde{r}_{C}(f)$, and in order to make it easier to see how these examples are related to some of the cases where one has equality, we state [4, Theorems 2.2 and 2.3] below (and give a short proof of the former result).

Here a cone $C$ in a real vector space $X$ is (following Wikipedia at the moment of writing but noting that often additional properties are required of a cone) defined to be a subset of $X$ such that $\lambda x \in C$ provided $x \in C$ and $\lambda>0$. A convex cone is

Received by the editors May 24, 2013 and, in revised form, August 12, 2013.

2010 Mathematics Subject Classification. Primary $47 \mathrm{H} 07$.

(C) 2014 American Mathematical Society Reverts to public domain 28 years from publication 
a cone $C$ in $X$ such that $C$ is a convex subset of $X$, and this is the case if and only if $x+y \in C$ for all $x$ and $y \in C$. If $X$ is e.g. a normed linear space, then a cone $C$ in $X$ is said to be complete (a complete cone) if it is a complete metric space in the topology induced by $X$. In the case where $X$ is a Banach space this is equivalent to $C$ being closed in $X$.

With the aid of a cone $C \subset X$ one can define relations $\leq_{C}$ and $\geq_{C}$ on $X$ so that $x \leq_{C} y$ or equivalently $y \geq_{C} x$ if and only if $y-x \in C$. These relations are reflexive if $0 \in C$, transitive if $C$ is convex, and anti-symmetric if $C \cap(-C) \subset\{0\}$ (where $-C=\{-x: x \in C\})$, and if all these conditions hold we have a partial order.

A function $f: C \rightarrow C$ is said to be order-preserving if $f(x) \leq_{C} f(y)\left(f(x) \geq_{C}\right.$ $f(y))$ whenever $x, y \in C$ and $x \leq_{C} y\left(x \geq_{C} y\right)$. Furthermore, it is said to be homogeneous of degree 1 if $f(\lambda x)=\lambda f(x)$ for all $\lambda>0$ and $x \in C$.

\section{TWO CASES WHERE $r_{C}(f)=\tilde{r}_{C}(f)$}

The following theorem is essentially [4, Theorem 2.2].

Theorem 2.1. Assume that

(i) $X$ is a real normed linear space.

(ii) $C$ is a convex and complete cone in $X$ such that $C \cap(-C)=\{0\}$.

(iii) $f: C \rightarrow C$ is order-preserving and homogeneous of degree 1 .

(iv) There is an integer $m \geq 1$ and a number $M<\infty$ such that if $x, y \in f^{m}(C)$ and $x \leq_{C} y$ then $\|x\|_{X} \leq M\|y\|_{X}$.

Then $r_{C}(f)=\tilde{r}_{C}(f)$.

When $f$ maps $C$ into $C$ it is clear that if $\|x\|_{X} \leq M\|y\|_{X}$ whenever $x \leq_{C} y$ and $x, y \in C$ (i.e., if the cone is normal), then hypothesis (iv) holds.

Proof of Theorem 2.1. Clearly we may assume that $C \neq\{0\}$. Then for each $k \geq 1$ there is a vector $x_{k} \in C$ with $\left\|x_{k}\right\|_{X}=1$ such that $\left\|f^{k}\left(x_{k}\right)\right\| \geq \frac{k}{k+1}\left\|f^{k}\right\|_{C}$ (or $\left\|f^{k}\left(x_{k}\right)\right\| \geq k^{k}$ if $\left.\left\|f^{k}\right\|_{C}=\infty\right)$. Since $C$ is a convex and complete cone we can construct a vector $x \in C$ by

$$
x=\sum_{j=1}^{\infty} j^{-2} x_{j} .
$$

We have $k^{-2} x_{k} \leq_{C} x$ for each $k \geq 1$ and since $f$, and hence $f^{k}$, is order-preserving and homogeneous of degree 1 we have

$$
k^{-2} f^{k}\left(x_{k}\right) \leq_{C} f^{k}(x), \quad k \geq 1 .
$$

Now it follows from assumption (iv) that

$$
\left\|f^{k}\left(x_{k}\right)\right\|_{X}^{1 / k} \leq\left(k^{2} M\right)^{1 / k}\left\|f^{k}(x)\right\|_{X}^{1 / k}, \quad k \geq m .
$$

This inequality together with our choice of $x_{k}$ and the definitions of $\tilde{r}_{c}(f)$ and $r_{C}(f)$ imply that

$$
\tilde{r}_{C}(f) \leq \limsup _{k \rightarrow \infty}\left\|f^{k}\left(x_{k}\right)\right\|_{X}^{1 / k} \leq \limsup _{k \rightarrow \infty}\left(k^{2} M\right)^{1 / k}\left\|f^{k}(x)\right\|_{X}^{1 / k} \leq r_{C}(f),
$$

which gives the claim as one always has $r_{C}(f) \leq \tilde{r}_{C}(f)$.

For completeness we include (a slight reformulation) of [4, Theorem 2.3] where the main assumption is that $f^{m}$ is compact. For the proof, see [4]. 
Theorem 2.2. Assume that

(i) $X$ is a real normed linear space.

(ii) $C$ is a nonempty cone in $X$.

(iii) $f: C \rightarrow C$ is continuous and homogeneous of degree 1 .

(iv) There is an integer $m \geq 1$ such that the closure of $f^{m}(B)$ is a compact subset of $C$ (in the topology induced by $X$ ) for every bounded subset $B$ of C.

Then $r_{C}(f)=\tilde{r}_{C}(f)$.

\section{EXAMPLES WHERE $r_{C}(f)<\tilde{r}_{C}(f)$}

The main example involves the case where hypothesis (iv) in Theorem 2.1 (and in Theorem 2.2) does not hold.

Example 3.1. Here we give an example of a Banach space $X$, a closed and convex cone $C \subset X$ such that $C \cap(-C)=\{0\}$, and a function $f: C \rightarrow C$ which is continuous, order-preserving, and homogeneous of degree 1 such that $r_{C}(f)<\tilde{r}_{C}(f)$.

Write points $V \in \mathbb{R}^{2}$ as $(x(V), y(V))$ and let $X=\left\{U: \mathbb{Z}_{+} \rightarrow \mathbb{R}^{2}:\|U\|_{X}<\infty\right\}$ where

$$
\|U\|_{X}=\sum_{n=1}^{\infty}\left(2^{n}|x(U(n))|+|x(U(n))+y(U(n))|\right) .
$$

For every $\alpha>0$ we define a closed and convex cone $C_{\alpha}$ in $\mathbb{R}^{2}$ by

$$
C_{\alpha}=\left\{V \in \mathbb{R}^{2}: x(V)+y(V) \geq \alpha \max \{0, x(V)\}\right\},
$$

and then we can define a closed and convex cone $C$ in $X$ by

$$
C=\left\{U \in X: U(n) \in C_{2^{-n}}, n \geq 1\right\} .
$$

Note also that with this definition we have $C \cap-C=\{0\}$ because $C_{\alpha} \cap\left(-C_{\alpha}\right)=\{0\}$ for all $\alpha>0$.

Let $h:[0, \infty) \times \mathbb{R}^{2} \rightarrow \mathbb{R}^{2}$ be the continuous function

$$
h(q, V)=(q x(V), y(V)+(1-q) x(V)),
$$

and let $g:[0, \infty) \times \mathbb{R}^{2} \rightarrow \mathbb{R}^{2}$ be the continuous function

$$
g(q, V)=(\min \{x(h(q, V)), x(V)+y(V)\}, \max \{y(h(q, V)), 0\}) .
$$

Note that both of these functions leave the sum of the coordinates of the point $V$ unchanged and that when $V=U(n)$ with $U \in C$ this sum is non-negative.

We define the function $q$ on $C$ as follows: If $U \in C$ and $\sum_{k=1}^{\infty}(x(U(k))+$ $y(U(k)))=0$ then $q(U)(n)=0$ for all $n \geq 1$ and otherwise

$$
q(U)(n)=\frac{\max \{0, x(U(n))\}}{\sum_{j=1}^{\infty}(x(U(j))+y(U(j)))}, \quad n \geq 1 .
$$

Now we can define the function $f$ on $C$ as follows:

$$
f(U)(n+1)= \begin{cases}(0,0), & \text { if } n=2^{p}-1, p \geq 0 \\ \frac{1}{2} g(q(U)(n), U(n)), & \text { for all other } n \geq 1\end{cases}
$$

We formulate the claims that this is an example with the desired properties in a series of lemmas. The proof of the first lemma is left to the reader. 
Lemma 3.2. Let $X, C$, and $f$ be as in Example 3.1 above. Then $X$ is a Banach space, $C$ is a closed, convex cone such that $C \cap(-C)=\{0\}$ and $f: C \rightarrow X$ is homogeneous of degree 1 .

The next lemma is the crucial part of verification of the example.

Lemma 3.3. Let $X, C$, and $f$ be as in Example 3.1 above. Then $f$ is orderpreserving and $f(C) \subset C$.

Proof. Suppose $U_{1}, U_{2} \in C$ with $U_{1} \geq_{C} U_{2}$. Let $n \geq 1$, write $q_{j}=q\left(U_{j}\right)$, $U_{j}(n)=\left(x_{j}, y_{j}\right)$ and $g\left(q_{j}, U_{j}(n)\right)=\left(\hat{x}_{j}, \hat{y}_{j}\right)$ for $j=1,2$. If we can prove that $\left(\hat{x}_{1}, \hat{y}_{1}\right) \geq_{C_{2-n-1}}\left(\hat{x}_{2}, \hat{y}_{2}\right)$ then we get the claim that $f$ is order-preserving.

Note that it follows from the assumption $\left(x_{1}, y_{1}\right) \geq_{C^{2-n}}\left(x_{2}, y_{2}\right)$ and the definitions of $h$ and $g$ that

$$
\begin{aligned}
\hat{x}_{1}+\hat{y}_{1}=x\left(h\left(q_{1}, U_{1}(n)\right)\right)+y\left(h\left(q_{1}, U_{1}(n)\right)\right)=x_{1}+y_{1} \\
\geq x_{2}+y_{2}=x\left(h\left(q_{2}, U_{2}(n)\right)\right)+y\left(h\left(q_{2}, U_{2}(n)\right)\right)=\hat{x}_{2}+\hat{y}_{2} .
\end{aligned}
$$

First we suppose that $\min \left\{x_{1}, x_{2}\right\} \leq 0$. If $x_{1} \leq 0$, then we have $0=\hat{x}_{1} \leq \hat{x}_{2}$, that is, $\max \left\{0, \hat{x}_{1}-\hat{x}_{2}\right\}=0$ which by (3.1) implies that $\left(\hat{x}_{1}, \hat{y}_{1}\right) \geq_{C_{2-n-1}}\left(\hat{x}_{2}, \hat{y}_{2}\right)$. If on the other hand $x_{1}>0$, which implies that $x_{2} \leq 0$, then $\hat{x}_{1} \leq x_{1}$ since the only case where $q_{1}>1$ is where $y_{1}<0$ and then $\hat{x}_{1}=x_{1}+y_{1}<x_{1}$. Thus we have $\hat{x}_{1}-\hat{x}_{2} \leq x_{1}-x_{2}$ and since by assumption $x_{1}-x_{2}+y_{1}-y_{2} \geq 2^{-n} \max \left\{0, x_{1}-x_{2}\right\}$ we get by (3.1) that $\hat{x}_{1}-\hat{x}_{2}+\hat{y}_{1}-\hat{y}_{2} \geq 2^{-n-1} \max \left\{0, \hat{x}_{1}-\hat{x}_{2}\right\}$, that is $\left(\hat{x}_{1}, \hat{y}_{1}\right) \geq_{C_{2-n-1}}$ $\left(\hat{x}_{2}, \hat{y}_{2}\right)$.

Suppose next that $x_{j}>0$ for $j=1,2$. It follows that $q_{1}$ and $q_{2}$ are positive as well. First we show that

$$
h\left(q_{1},\left(x_{1}, y_{1}\right)\right) \geq_{C_{2-n-1}} h\left(q_{2},\left(x_{2}, y_{2}\right)\right) .
$$

If $q_{1} x_{1}-q_{2} x_{2} \leq 0$, then we recall that by (3.1) we have $x_{1}+y_{1} \geq x_{2}+y_{2}$ and hence we get

$$
\begin{aligned}
q_{1} x_{1}-q_{2} x_{2}+y_{1}+\left(1-q_{1}\right) x_{1}-\left(y_{2}+\left(1-q_{2}\right) x_{2}\right) & =x_{1}+y_{1}-\left(x_{2}+y_{2}\right) \geq 0 \\
& =2^{-n-1} \max \left\{0, q_{1} x_{1}-q_{2} x_{2}\right\}
\end{aligned}
$$

which is the claim (3.2).

Suppose next that $q_{1} x_{1}-q_{2} x_{2}>0$. Since $U_{1} \geq_{C} U_{2}$ implies that $\sum_{j=1}^{\infty}\left(x\left(U_{1}(j)\right)+\right.$ $\left.y\left(U_{1}(j)\right)\right) \geq \sum_{j=1}^{\infty}\left(x\left(U_{2}(j)\right)+y\left(U_{2}(j)\right)\right)$ we have

$$
\frac{q_{1}}{x_{1}} \leq \frac{q_{2}}{x_{2}} .
$$

This inequality combined with $q_{1} x_{1}-q_{2} x_{2}>0$ implies, since $x_{1}$ and $q_{1}>0$, that

$$
x_{1}>\frac{q_{2} x_{2}}{q_{1}} \geq \frac{x_{2}^{2}}{x_{1}} \text {. }
$$

Thus $x_{1}^{2}>x_{2}^{2}$ which implies that

$$
x_{1}>x_{2}>0,
$$

since both numbers are assumed to be positive. Using inequality (3.3) and the positivity of $x_{2}$ once more we obtain

$$
\left(q_{1} x_{1}-q_{2} x_{2}\right) \leq q_{1}\left(x_{1}-\frac{x_{2}^{2}}{x_{1}}\right)=q_{1} \frac{x_{1}+x_{2}}{x_{1}}\left(x_{1}-x_{2}\right) .
$$


Provided we assume for the moment that

$$
q_{1} \frac{x_{1}+x_{2}}{x_{1}} \leq 2
$$

we can conclude that

$$
2^{-n}\left(x_{1}-x_{2}\right) \geq 2^{-n-1}\left(q_{1} x_{1}-q_{2} x_{2}\right) .
$$

The inequalities $\left(x_{1}, y_{1}\right) \geq_{C_{2-n}}\left(x_{2}, y_{2}\right)$ and (3.4) imply that

$$
x_{1}-x_{2}+y_{1}-y_{2} \geq 2^{-n}\left(x_{1}-x_{2}\right),
$$

and when this inequality is combined with (3.6) and the assumption $q_{1} x_{1}-q_{2} x_{2}>0$ we get

$$
\begin{aligned}
q_{1} x_{1}-q_{2} x_{2}+y_{1}+\left(1-q_{1}\right) x_{1}-\left(y_{2}+\left(1-q_{2}\right) x_{2}\right) & =x_{1}-x_{2}+y_{1}-y_{2} \\
& \geq 2^{-n}\left(x_{1}-x_{2}\right) \geq 2^{-n-1}\left(q_{1} x_{1}-q_{2} x_{2}\right)=2^{-n-1} \max \left\{0, q_{1} x_{1}-q_{2} x_{2}\right\} .
\end{aligned}
$$

This is the claim (3.2).

If (3.2) holds (recall that we needed assumption (3.5) in case $q_{1} x_{1}-q_{2} x_{2}>0$ ), then $\left(\hat{x}_{1}, \hat{y}_{1}\right) \geq_{C_{2-n-1}}\left(\hat{x}_{2}, \hat{y}_{2}\right)$ in the case where $\hat{x}_{2}=x\left(h\left(q_{2}, U_{2}\right)\right)$ because $\hat{x}_{1} \leq$ $x\left(h\left(q_{1}, U_{1}\right)\right)$ and then it follows from (3.1) that

$$
\begin{aligned}
& 2^{-n-1} \max \left\{0, \hat{x}_{1}-\hat{x}_{2}\right\} \leq 2^{-n-1} \max \left\{0, x\left(h\left(q_{1}, U_{1}\right)\right)-x\left(h\left(q_{2}, U_{2}\right)\right)\right\} \\
\leq & x\left(h\left(q_{1}, U_{1}\right)\right)-x\left(h\left(q_{2}, U_{2}\right)\right)+y\left(h\left(q_{1}, U_{1}\right)\right)-y\left(h\left(q_{2}, U_{2}\right)\right)=\hat{x}_{1}-\hat{x}_{2}+\hat{y}_{1}-\hat{y}_{2} .
\end{aligned}
$$

If on the other hand $\hat{x}_{2}<x\left(h\left(q_{2}, U_{2}\right)\right)$, then $0=\hat{y}_{2} \leq \hat{y}_{1}$ and then we conclude in the case where $\hat{x}_{1}>\hat{x}_{2}$ that

$$
2^{-n-1} \max \left\{0, \hat{x}_{1}-\hat{x}_{2}\right\}<\hat{x}_{1}-\hat{x}_{2} \leq \hat{x}_{1}-\hat{x}_{2}+\hat{y}_{1}-\hat{y}_{2},
$$

and in the case where $\hat{x}_{1} \leq \hat{x}_{2}$ we conclude by (3.1) that

$$
2^{-n-1} \max \left\{0, \hat{x}_{1}-\hat{x}_{2}\right\}=0 \leq x_{1}-x_{2}+y_{1}-y_{2}=\hat{x}_{1}-\hat{x}_{2}+\hat{y}_{1}-\hat{y}_{2} .
$$

Hence we have $\left(\hat{x}_{1}, \hat{y}_{1}\right) \geq_{C^{2}-n-1}\left(\hat{x}_{2}, \hat{y}_{2}\right)$ in the case where $\hat{x}_{2}<x\left(h\left(q_{2}, U_{2}\right)\right)$ as well.

Thus it remains to consider the case where $q_{1} x_{1}-q_{2} x_{2}>0$ and hence (3.4) holds but (3.5) does not hold. If $\hat{y}_{2}=0$, then $\hat{y}_{2} \leq \hat{y}_{1}$ and the claim follows by inequalities (3.8) and (3.9) as above. If $\hat{y}_{2}>0$, then we have $q_{2} \leq 1$ and $\hat{x}_{2}=q_{2} x_{2}$. If $q_{1} \leq 1$, then it follows from (3.4) that (3.5) holds, and since we assumed that this is not the case we must have $q_{1}>1$ and therefore $y_{1}<0$ so that $\hat{x}_{1}=x_{1}+y_{1}$ and $\hat{y}_{1}=0$. Thus we have to show that

$$
x_{1}+y_{1}-q_{2} x_{2}+0-\left(y_{2}+\left(1-q_{2}\right) x_{2}\right) \geq 2^{-n-1}\left(x_{1}+y_{1}-q_{2} x_{2}\right),
$$

or, equivalently

$$
x_{1}-x_{2}+y_{1}-y_{2}-2^{-n-1}\left(x_{1}+y_{1}-q_{2} x_{2}\right) \geq 0 .
$$

Since inequality (3.7) holds in this case as well we get (3.10) provided

$$
2^{-n}\left(x_{1}-x_{2}\right)-2^{-n-1}\left(x_{1}+y_{1}-q_{2} x_{2}\right) \geq 0 .
$$

Since $y_{1}<0$ this inequality holds provided

$$
x_{1}-\left(2-q_{2}\right) x_{2} \geq 0 \text {. }
$$


But we assumed that (3.5) does not hold, which by (3.3) and (3.4) implies that

$$
q_{2} \frac{x_{1}+x_{2}}{x_{2}}>2
$$

and (3.11) follows from this inequality because $q_{2} \leq 1$ in this case as noted above. This completes the proof of the fact that $f$ is order-preserving.

Since $0 \in C, f(0)=0$, and $f$ is order-preserving it follows that $f$ maps $C$ into itself.

Lemma 3.4. Let $X, C$, and $f$ be as in Example 3.1 above. Then $f$ is continuous.

Proof. First we note that since the mapping $g$ does not increase the absolute value of the $x$-coordinate and leaves the sum of the coordinates unchanged, it follows from the definition of $f$ that

$$
\left|x\left(f\left(U_{j}\right)(n+1)\right)\right| \leq \frac{1}{2}\left|x\left(U_{j}(n)\right)\right|, \quad n \geq 1,
$$

and

$$
\begin{gathered}
\left|x\left(f\left(U_{j}\right)(n+1)\right)+y\left(f\left(U_{j}\right)(n+1)\right)-(x(f(U)(n+1))+y(f(U)(n+1)))\right| \\
\leq \frac{1}{2}\left|x\left(U_{j}(n)\right)+y\left(U_{j}(n)\right)-(x(U(n))+y(U(n)))\right|, \quad n \geq 1 .
\end{gathered}
$$

Now assume that $U_{j} \in C, j \geq 1$, are such that $\lim _{j \rightarrow \infty}\left\|U_{j}-U\right\|_{X}=0$ for some $U \in C$ or equivalently,

$$
\lim _{j \rightarrow \infty} \sum_{n=1}^{\infty} 2^{n}\left|x\left(U_{j}(n)\right)-x(U(n))\right|=0,
$$

and

$$
\lim _{j \rightarrow \infty} \sum_{n=1}^{\infty}\left|x\left(U_{j}(n)\right)+x\left(U_{j}(n)\right)-(x(U(n))+y(U(n)))\right|=0 .
$$

First we consider the case where $\sum_{n=1}^{\infty}(x(U(n))+y(U(n)))=0$. Since $U \in C$ we have $x(U(n))+y(U(n))=0$ and $x(U(n)) \leq 0$ for all $n$ and therefore $f(U)=0$. By (3.15) $\lim _{j \rightarrow \infty} \sum_{n=1}^{\infty}\left(x\left(U_{j}(n)\right)+y\left(U_{j}(n)\right)\right)=0$ and hence by (3.13) we obtain

$$
\lim _{j \rightarrow \infty} \sum_{n=1}^{\infty}\left(x\left(f\left(U_{j}\right)(n)\right)+y\left(f\left(U_{j}\right)(n)\right)\right)=0 .
$$

Since $\lim _{j \rightarrow \infty} x\left(U_{j}(n)\right)=x(U(n)) \leq 0$ for each $n \geq 1$ we get $\lim _{j \rightarrow \infty} x\left(f\left(U_{j}\right)(n)\right)=$ 0 for each $n \geq 1$ and this result together with (3.14) and (3.12) implies that $\lim _{j \rightarrow \infty} \sum_{n=1}^{\infty} 2^{n}\left|x\left(f\left(U_{j}\right)(n)\right)\right|=0$. When this result is combined with (3.16) we get $\lim _{j \rightarrow \infty} f\left(U_{j}\right)=0=f(U)$ in $X$ in the case where $\sum_{n=1}^{\infty}(x(U(n))+y(U(n)))=0$.

Suppose next that $\sum_{n=1}^{\infty}(x(U(n))+y(U(n)))>0$. Then it follows from the defintion of $q$ and (3.15) that

$$
\lim _{j \rightarrow \infty} \sup _{n \geq 1}\left|q\left(U_{j}\right)(n)-q(U)(n)\right|=0,
$$

and this implies in turn that

$$
\lim _{j \rightarrow \infty} \sup _{n \geq 1}\left|x\left(f\left(U_{j}\right)(n)\right)-x(f(U)(n))\right|=0 .
$$


Combining this fact with (3.12) and (3.14) we get

$$
\lim _{j \rightarrow \infty} \sum_{n=1}^{\infty} 2^{n}\left|x\left(f\left(U_{j}\right)(n)\right)-x(f(U)(n))\right|=0 .
$$

Since it follows from (3.13) and (3.15) that

$$
\lim _{j \rightarrow \infty} \sum_{n=1}^{\infty}\left|x\left(f\left(U_{j}\right)(n)\right)+y\left(f\left(U_{j}\right)(n)\right)-(x(f(U)(n))+y(f(U)(n)))\right|=0 .
$$

we get the desired conclusion $\lim _{j \rightarrow \infty} f\left(U_{j}\right)=f(U)$ in $X$ in this case as well. This completes the proof of the continuity of $f$ on $C$.

Finally we turn to the estimation of the cone spectral radii.

Lemma 3.5. Let $X, C$, and $f$ be as in Example 3.1. Then $\tilde{r}_{C}(f) \geq 1$ and $r_{C}(f) \leq$ $\frac{1}{2}$.

Proof. First we show that $\tilde{r}_{C}(f) \geq 1$. Let $k \geq 1$, choose $j \geq 1$ such that $2^{j-1}>k$, and take

$$
U(n)= \begin{cases}\left(2^{-n}, 0\right), & n=2^{j-1} \\ (0,0), & \text { for all other } n \geq 1\end{cases}
$$

Thus $\|U\|_{X}=1+2^{-2^{j-1}}$ and

$$
f^{k}(U)(n)= \begin{cases}\left(2^{-n-k}, 0\right), & n=2^{j-1}+k \\ (0,0), & \text { for all other } n \geq 1\end{cases}
$$

so that $\left\|f^{k}(U)\right\|_{X}=1+2^{-2^{j-1}-k}$. Since $j$ can be chosen arbitrarily large we conclude that $\left\|f^{k}\right\|_{C} \geq 1$ and hence $\tilde{r}_{C}(f) \geq 1$.

Next we show that $r_{C}(f) \leq \frac{1}{2}$ and first we note that if $U(n)=(0,0)$, then $f(U)(n+1)=(0,0)$ and thus it follows from the fact that $f(U)\left(2^{p}\right)=(0,0)$ for every $p \geq 0$ that $f^{k}(U)(n)=(0,0)$ when $k \geq 1$ and $2^{p} \leq n<2^{p}+k$. Therefore

$$
f^{k}(U)(n)=(0,0), \quad 1 \leq n<2 k, \quad k \geq 1,
$$

for all $U \in C$.

It follows from the definitions of $q$ and $f$ that there is an integer $n_{U} \geq 1$ such that $q(U)(n) \leq \frac{1}{2}$ and $q(f(U))(n) \leq \frac{1}{2}$ when $n \geq n_{U}$. Hence $|x(f(U)(n+1))| \leq$ $\frac{1}{4}|x(U(n))|$ when $n \geq n_{U}$ and we have $|x(f(U)(n+1))+y(f(U)(n+1))| \leq$ $\frac{1}{2}|x(U(n))+y(U(n))|$ for all $n \geq 1$. Furthermore we have $q\left(f^{k}(U)\right)(n) \leq \frac{1}{2}$ when $n \geq n_{U}+k$ so we have in fact

$$
\mid x\left(f^{k}(U)(n+k)\left|\leq 4^{-k}\right| x(U(n)) \mid, \quad n \geq n_{U},\right.
$$

and

$$
\left|x\left(f^{k}(U)(n+k)\right)+y\left(f^{k}(U)(n+k)\right)\right| \leq 2^{-k}|x(U(n))+y(U(n))|, \quad n \geq 1 .
$$

Combined with (3.17) these inequalities show that $\lim _{\sup } \operatorname{sum}_{k \rightarrow \infty} 2^{k}\left\|f^{k}(U)\right\|_{X} \leq\|U\|_{X}$ and hence $r_{C}(f) \leq \frac{1}{2}$.

We give four further examples where $r_{C}(f)=0$ and $\tilde{r}_{C}(f)=1$ but observe that these examples are not that significant since each hypothesis that is dropped is quite reasonable (and e.g. the convexity assumption is often included in the definition of a cone). 
In all of these examples we take $X=c_{0}=\left\{U: \mathbb{Z}_{+} \rightarrow \mathbb{R}: \lim _{n \rightarrow \infty} U(n)=0\right\}$ with norm $\|U\|_{X}=\sup _{n \geq 1}|U(n)|$ and let $C$ be a subset of the cone $\left\{U \in c_{0}\right.$ : $U(n) \geq 0, n \geq 1\}$ in $c_{0}$. Hence $C$ is a normal cone (if $x, y \in C, x \leq_{C} y$ then $\|x\|_{X} \leq M\|y\|_{X}$ for some $M<\infty$, in this case $M=1$ ) and hypothesis (iv) of Theorem 2.1 holds. In addition it follows that we have $C \cap(-C)=\{0\}$ in all these examples. In the first three of these examples the function $f$ is continuous, an assumption made in [4, Theorem 2.2] but not in Theorem 2.1.

In the first example the cone $C$ is not convex but all other hypotheses of Theorem 2.1 are satisfied.

Example 3.6. Let $X=c_{0}$,

$$
C=\left\{U \in c_{0}: U(n)=U(1) \geq 0,1 \leq n \leq n_{U}, \quad U(n)=0, n>n_{U} \geq 1\right\},
$$

and define $f: C \rightarrow C$ by $f(U)(n)=U(n+1), n \geq 1$.

In the second example the cone $C$ is not closed in $X$ (hence not complete) but all other hypotheses of Theorem 2.1 are satisfied.

Example 3.7. Let $X=c_{0}$,

$$
C=\left\{U \in c_{0}: U(n) \geq 0, n \geq 1, \quad U(n)=0, \quad n \geq n_{U} \geq 1\right\},
$$

and define $f: C \rightarrow C$ by $f(U)(n)=U(n+1), n \geq 1$.

In the third example the function $f$ is not order-preserving but all other hypotheses of Theorem 2.1 are satisfied. A similar example is given on page 526 in [4.

Example 3.8. Let $X=c_{0}$,

$$
C=\left\{U \in c_{0}: U(n) \geq 0, n \geq 1\right\},
$$

and define $f: C \rightarrow C$ by $f(0)=0$ and

$$
f(U)(n)=U(n+1)\left(\frac{U(n+1)}{\sup _{j \geq 1} U(j)}\right)^{n}, \quad n \geq 1,
$$

when $U \neq 0$.

In the final example the function $f$ is not continuous, nor order-preserving, but all other hypotheses of both Theorems 2.2 and 2.1 are satisfied.

Example 3.9. Let $X=c_{0}$,

$$
C=\left\{U \in c_{0}: U(n) \geq 0, n \geq 1\right\},
$$

and define $f$ by

$$
f(U)(n)= \begin{cases}2 U(n+1), & U \in C_{f}, \quad n \geq 1, \\ 0, & \text { otherwise }\end{cases}
$$

where

$C_{f}=\left\{U \in C: U(n+1)=\frac{1}{2} U(n), 1 \leq n \leq n_{U}-1, \quad U(n)=0, n>n_{U} \geq 1\right\}$.

\section{ACKNOWLEDGEMENT}

The author thanks the anonymous referee for several suggestions to improve the presentation in this paper. 


\section{REFERENCES}

[1] Marianne Akian, Stéphane Gaubert, and Roger Nussbaum, A Collatz-Wielandt characterization of the spectral radius of order-preserving homogeneous maps on cones, arXive:1112.5968v1, 2011.

[2] F. F. Bonsall, Linear operators in complete positive cones, Proc. London Math. Soc. (3) 8 (1958), 53-75. MR0092938 (19,1183c)

[3] Bas Lemmens and Roger Nussbaum, Continuity of the cone spectral radius, Proc. Amer. Math. Soc. 141 (2013), no. 8, 2741-2754, DOI 10.1090/S0002-9939-2013-11520-0. MR3056564

[4] John Mallet-Paret and Roger D. Nussbaum, Eigenvalues for a class of homogeneous cone maps arising from max-plus operators, Discrete Contin. Dyn. Syst. 8 (2002), no. 3, 519-562, DOI 10.3934/dcds.2002.8.519. MR.1897866 (2003c:47088)

[5] John Mallet-Paret and Roger D. Nussbaum, Generalizing the Krein-Rutman theorem, measures of noncompactness and the fixed point index, J. Fixed Point Theory Appl. 7 (2010), no. 1, 103-143, DOI 10.1007/s11784-010-0010-3. MR2652513(2011j:47148)

Department of Mathematics and Systems Analysis, Aalto University, VÄrmEMANSGRÄNDEN 2, 02150 ESPOO, Finland

E-mail address: gustaf.gripenberg@aalto.fi 